University of Texas at El Paso

ScholarWorks@UTEP

$6-2000$

\title{
Towards Reliable Sub-Division of Geological Areas: Interval Approach
}

\author{
David D. Coblentz \\ Vladik Kreinovich \\ The University of Texas at El Paso, vladik@utep.edu \\ Brian S. Penn \\ Scott A. Starks \\ The University of Texas at El Paso, sstarks@utep.edu
}

Follow this and additional works at: https://scholarworks.utep.edu/cs_techrep

Part of the Computer Engineering Commons

\section{Comments:}

UTEP-CS-00-24a.

Published in Proceedings of the 19th International Conference of the North American Fuzzy Information Society NAFIPS'2000, Atlanta, Georgia, July 13-15, 2000, pp. 368-372; extended version published in: L. Reznik and V. Kreinovich (eds.), Soft Computing in Measurements and Information Acquisition, Springer-Verlag, 2003, pp. 223-233.

\section{Recommended Citation}

Coblentz, David D.; Kreinovich, Vladik; Penn, Brian S.; and Starks, Scott A., "Towards Reliable Sub-Division of Geological Areas: Interval Approach" (2000). Departmental Technical Reports (CS). 481.

https://scholarworks.utep.edu/cs_techrep/481

This Article is brought to you for free and open access by the Computer Science at ScholarWorks@UTEP. It has been accepted for inclusion in Departmental Technical Reports (CS) by an authorized administrator of ScholarWorks@UTEP. For more information, please contact Iweber@utep.edu. 


\title{
Towards Reliable Sub-Division of Geological Areas: Interval Approach
}

\author{
David D. Coblentz ${ }^{1,2}$, Vladik Kreinovich ${ }^{1}$, Brian S. Penn ${ }^{1,3}$, and \\ Scott A. Starks ${ }^{1}$ \\ 1 NASA Pan American Center for Earth and Environmental Studies, \\ University of Texas, El Paso TX 79968, USA, contact email vladik@cs.utep.edu \\ 2 Institut für Geologie und Paläontologie, Universität Graz, Heinrichstr 26, \\ A-8010 Graz, Austria \\ 3 Autometric, Inc., 1330 Inverness Drive, Suite 350, Colorado Springs, CO 80910, \\ USA
}

\begin{abstract}
An appropriate subdivision of a geophysical area into segments enables us to extrapolate the results obtained in some locations within the segment (where extensive research was done) to other locations within the same segment, and thus, get a good understanding of the locations which weren't thoroughly analyzed.

Often, different evidence and different experts' intuition support different subdivisions schemes. For example, in our area - Rio Grande rift zone - there is some geochemical evidence that this zone is divided into three segments, but, in the viewpoint of many researchers, this evidence is not yet sufficiently convincing.

We show that if we use topographical information (this information, e.g., comes from satellite photos), then interval methods lead to a reliable justification for the tripartite subdivision of the Rio Grande rift zone.
\end{abstract}

\section{Appropriate Subdivision Is Important In Geophysics}

In geophysics, appropriate subdivision of an area into segments is extremely important, because it enables us to extrapolate the results obtained in some locations within the segment (where extensive research was done) to other locations within the same segment, and thus, get a good understanding of the locations which weren't that thoroughly analyzed.

\section{A Problem: It Is Often Difficult to Produce a Reliable Subdivision}

The subdivision of a geological zone into segments is often a controversial issue, with different evidence and different experts' intuition supporting different subdivisions.

For example, in our area - Rio Grande rift zone - there is some geochemical evidence that this zone is divided into three segments [5]: 
- the southern segment which is located, approximately, between the latitudes $y=29^{\circ}$ and $y=34^{\circ}$;

- the central segment - from $y=34.5^{\circ}$ to $y=38^{\circ}$; and

- the northern segment - from $y=38^{\circ}$ to $y=41^{\circ}$.

However, in the viewpoint of many researchers, this evidence is not yet sufficiently convincing.

It is therefore desirable to develop new techniques for zone sub-division, techniques which would be in the least possible way dependent on the (subjective) expert opinion and would, thus, be maximally reliable.

\section{Main Idea: Using Topographic Information}

One reason for subjectivity is the fact that the existing subdivision is often based on the chemical and physical analysis of several samples collected throughout the area, and often, we do not have a statistically sufficient amount of thoroughly analyzed geological samples to make the conclusion about the subdivision statistically convincing.

To make this conclusion more reliable, we can use, instead of the more rare geological samples, a more abundant topographical information (this information, e.g., comes from satellite photos). We can characterize each part of the divided zone by its topography.

\section{Preserving Only Geophysically Meaningful Topographic Information: The Use of Spectral Values Corresponding to Long Wavelengths}

In topographical analysis, we face a new problem: of too much data, most of which is geophysically irrelevant. To eliminate some of this irrelevant data, we can use the Fourier transform; indeed, it is known that while (at least some) absolute values of the map (forming a so-called spectrum) are geophysically meaningful, the phases usually are random and can be therefore ignored. So, we should only use the spectrum.

Since we are interested only in the large-scale classification, it makes sense to only use the spectrum values corresponding to relatively large spatial wavelengths, i.e., wavelengths $L$ for which $L \geq L_{0}$ for some appropriate value $L_{0}$. In particular, for the sub-division of the Rio Grande rift, it makes sense to use only wavelengths of $L_{0}=1000 \mathrm{~km}$ or larger.

Also, for the Rio Grande Rift, we are interested in the classification of horizontal zones, so it makes sense to do the following: 
- divide the Rio Grande Rift into $1^{\circ}$ zones $\left[y^{-}, y^{+}\right]$(with $y$ from $y^{-}=30$ to $y^{+}=31$, from $y^{-}=31$ to $y^{+}=32, \ldots$, from $y^{-}=40$ to $y^{+}=41$ );

- for each of these zones, take the topographic data, i.e., the height $h(x, y)$ described as a function of longitude $x$ and latitude $y$;

- for each zone and for each $y$, compute the Fourier transform $H(\omega, y)$ with respect to $x$;

- for each zone, combine all the spectral values which correspond to large wavelength (i.e., for which $\omega \leq 1 / L_{0}$ ), and compute the resulting spectral value

$$
S\left(y^{-}\right)=\int_{y=y^{-}}^{y^{+}} \int_{\omega=0}^{1 / L_{0}}|H(\omega, y)|^{2} \mathrm{~d} \omega \mathrm{d} y .
$$

We are interested in comparing the spectral values $S(y)$ corresponding to different latitudes $y$, so we are not interested in the absolute values of $S(y)$, only in relative values. Thus, to simplify the data, we can normalize them by, e.g., dividing each value $S\left(y^{-}\right)$by the largest $S_{\max }$ of these values. In particular, for the Rio Grande rift, the resulting values of $y^{-}=y_{1}, y_{2}, \ldots$ and $s_{i}=S\left(y_{i}\right) / S_{\max }$ are as follows:

Table 1.

\begin{tabular}{|c|c|c|c|c|c|c|}
\hline$y_{i}$ & 29 & 30 & 31 & 32 & 33 & 34 \\
\hline$s_{i}$ & 0.28 & 0.24 & 0.21 & 0.16 & 0.20 & 0.29 \\
\hline \hline 35 & 36 & 37 & 38 & 39 & 40 & 41 \\
\hline 0.31 & 0.35 & 0.46 & 1.00 & 0.80 & 0.96 & 0.74 \\
\hline
\end{tabular}

Based only on these spectral values $s_{i}$, we will try to classify locations into several clusters ("segments").

\section{Traditional Clustering Techniques Do Not Lead to Reliable Classification}

Most existing clustering methods (see, e.g., [2]) assume that we have some information about the probability distributions of the measurement errors. In real-life topographical analysis, we do not have enough data to uniquely determine these distributions, and different possible distributions lead to drastically different conclusions about the subdivision of a geological zone.

Similarly, fuzzy clustering techniques (see, e.g., [1]) depend on the subjective fuzzy estimates, and different expert estimates often lead to drastically different subdivisions.

Therefore, we need a new, less subjective clustering method. 


\section{Segments as Monotonicity Regions}

How can we detect the segments based on these values $s_{i}$ ? In order to answer this question, let us first plot the dependence of $s_{i}$ on $y_{i}$ and see if there is any visible feature of this plot which can be associated with a subdivision of the area into geophysical segments.

If we plot the dependence of $s_{i}$ on $y_{i}$, we will see that at first, the function $s(y)$ is (approximately) decreasing, then it is (approximately) increasing, and then it is (approximately) decreasing again. Interestingly, these "monotonicity regions" seem to be in good accordance with the empirical subdivision of the rift into segments [5]. In view of this observation, we will identify geophysical segments with the monotonicity regions of the (unknown) function $s(y)$.

\section{$7 \quad$ Let's Use Intervals}

The heights are measured pretty accurately, so the only errors in the values $s_{i}$ come from discretization. In other words, we would like to know the values of the function $s(y)=S(y) / S_{\max }$ for all $y$, but we only know the values $s_{1}=s\left(y_{1}\right), \ldots, s_{n}=s\left(y_{n}\right)$ of this function for the points $y_{1}, \ldots, y_{n}$. For each $y$ which is different from $y_{i}$, it is reasonable to estimate $s(y)$ as the value $s_{i}=s\left(y_{i}\right)$ at the point $y_{i}$ which is the closest to $y$ (and, ideally, which belongs to the same segment as $y_{i}$ ). For each point $y_{i}$, what is the largest possible error $\Delta_{i}$ of the corresponding approximation?

When $y>y_{i}$, the point $y_{i}$ is still the closest until we reach the midpoint $y_{\text {mid }}=\left(y_{i}+y_{i+1}\right) / 2$ between $y_{i}$ and $y_{i+1}$. It is reasonable to assume that the largest possible approximation error $\left|s(y)-s_{i}\right|$ for such points is attained when the distance between $y$ and $y_{i}$ is the largest, i.e., when $y$ is this midpoint; in this case, the approximation error is equal to $\left|s\left(y_{\text {mid }}\right)-s_{i}\right|$.

If the points $y_{i}$ and $y_{i+1}$ belong to the same segment, then the dependence of $s(y)$ on $y$ should be reasonably smooth for $y \in\left[y_{i}, y_{i+1}\right]$. Therefore, on a narrow interval $\left[y_{i}, y_{i+1}\right]$, we can, with reasonable accuracy, ignore quadratic and higher terms in the expansion of $s\left(y_{i}+\Delta y\right)$ and thus, approximate $s(y)$ by a linear function. For a linear function $s(y)$, the difference $s\left(y_{\text {mid }}\right)-s\left(y_{i}\right)$ is equal to the half of the difference $s\left(y_{i+1}\right)-s\left(y_{i}\right)=s_{i+1}-s_{i}$; thus, for $y>y_{i}$, the approximation error is bounded by $0.5 \cdot\left|s_{i+1}-s_{i}\right|$.

If the points $y_{i}$ and $y_{i+1}$ belong to different segments, then the dependence $s(y)$ should exhibit some non-smoothness, and it is reasonable to expect that the difference $\left|s_{i+1}-s_{i}\right|$ is much higher than the approximation error.

In both cases, the approximation error is bounded by

$$
0.5 \cdot\left|s_{i+1}-s_{i}\right| \text {. }
$$

Similarly, for $y<y_{i}$, the approximation error is bounded by $0.5 \cdot\left|s_{i}-s_{i-1}\right|$ if the points $y_{i}$ and $y_{i-1}$ belong to the same segment, and is much smaller if 
they don't. In both cases, the approximation error is bounded by

$$
0.5 \cdot\left|s_{i}-s_{i-1}\right| \cdot
$$

We have two bounds on the approximation error and we can therefore conclude that the approximation error cannot exceed the smallest $\Delta_{i}$ of these two bounds, i.e., the value

$$
\Delta_{i}=0.5 \cdot \min \left(\left|s_{i}-s_{i_{1}}\right|,\left|s_{i+1}-s_{i}\right|\right) .
$$

As a result, instead of the exact values $s_{i}$, for each $i$, we get the interval $\mathbf{s}_{i}=\left[s_{i}^{-}, s_{i}^{+}\right]$of possible values of $s(y)$, where $s_{i}^{-}=s_{i}-\Delta_{i}$ and $s_{i}^{+}=s_{i}+\Delta_{i}$. In particular, for the Rio Grande rift, the corresponding intervals are as follows:

Table 2.

\begin{tabular}{|c|c|c|c|c|}
\hline$y_{i}$ & $y_{1}=29$ & $y_{2}=30$ & $y_{3}=31$ & $y_{4}=32$ \\
\hline $\mathbf{s}_{i}=\left[s_{i}^{-}, s_{i}^{+}\right]$ & {$[0.26,0.30]$} & {$[0.225,0.255]$} & {$[0.195,0.225]$} & {$[0.14,0.18]$} \\
\hline \hline$y_{5}=33$ & $y_{6}=34$ & $y_{7}=35$ & $y_{8}=36$ & $y_{9}=37$ \\
\hline$[0.18,0.22]$ & {$[0.28,0.30]$} & {$[0.30,0.32]$} & {$[0.33,0.37]$} & {$[0.405,0.515]$} \\
\hline \hline$y_{10}=38$ & $y_{11}=39$ & $y_{12}=40$ & $y_{13}=41$ & \\
\hline$[0.80,1.10]$ & {$[0.72,0.88]$} & {$[0.88,1.04]$} & {$[0.63,0.85]$} \\
\hline
\end{tabular}

\section{How to Find Monotonicity Regions of a Function Defined With Interval Uncertainty: Idea}

We want to find regions of uncertainty of a function $s(y)$, but we do not know the exact form of this function; all we know is that for every $i, s\left(y_{i}\right) \in \mathbf{s}_{i}$ for known intervals $\mathbf{s}_{i}$. How can we find the monotonicity regions in the situation with such interval uncertainty?

Of course, since we only know the values of the function $s(y)$ in finitely many points $y_{i}$, this function can have as many monotonicity regions between $y_{i}$ and $y_{i+1}$ as possible. What we are interested in is funding the subdivision into monotonicity regions which can be deduced from the data. The first natural question is: can we explain the data by assuming that the dependence $s(y)$ is monotonic? If not, then we can ask for the possibility of having a function $s(y)$ with exactly two monotonicity regions: 
- if such a function is possible, then we are interested in possible locations of such regions;

- if such a function is not possible, then we will try to find a function $s(y)$ which is consisted with our interval data and which has three monotonicity regions, etc.

This problem was first formalized and solved in [8]. The corresponding algorithm is based on the following idea.

If the function $s(y)$ is non-decreasing, then, for $i<j$, we have $s\left(y_{i}\right) \leq$ $s\left(y_{j}\right)$; therefore, $s_{i}^{-} \leq s\left(y_{i}\right) \leq s\left(y_{j}\right) \leq s_{j}^{+}$and $s_{i}^{-} \leq s_{j}^{+}$. It turns out that, vice versa, if the inequality $s_{i}^{-} \leq s_{j}^{+}$hold for every $i<j$, then there is a non-decreasing function $s(y)$ for which $s\left(y_{i}\right) \in \mathbf{s}_{i}$ for all $i$ (e.g., we can take $s\left(y_{i}\right)=\max \left(s_{1}^{-}, \ldots, s_{i}^{-}\right)$for all $i$ and use linear interpolation to define the values $s(y)$ for $y \neq y_{i}$.) Thus, to check monotonicity, it is sufficient to check these inequalities.

If we have already checked these inequalities for the intervals $\mathbf{s}_{1}, \ldots, \mathbf{s}_{k}$, and we add the new interval $\mathbf{s}_{k+1}$, then, to confirm that it is still possible for a function $s(z)$ to be non-decreasing, it is sufficient to check that $s_{i}^{-} \leq s_{k+1}^{+}$ for all $i=1, \ldots, k$. These $k$ inequalities are equivalent to a single inequality $m_{k} \leq s_{k+1}^{+}$, where we denoted $m_{k}=\max \left(s_{1}^{-}, \ldots, s_{k}^{-}\right)$.

Similarly, in order to check that a function $s(y)$ can be non-increasing, it is sufficient to check that $s_{i}^{+} \geq s_{j}^{-}$for all pairs $i>j$. If we have already checked these inequalities for the intervals $\mathbf{s}_{1}, \ldots, \mathbf{s}_{k}$, and we add the new interval $\mathbf{s}_{k+1}$, then, to confirm that it is still possible for a function $s(z)$ to be non-increasing, it is sufficient to check that $M_{k} \geq s_{k+1}^{-}$, where we denoted $M_{k}=\min \left(s_{1}^{+}, \ldots, s_{k}^{+}\right)$.

The values $m_{k}$ and $M_{k}$ needed for these comparisons do not to be re-computed for every $k$; if we have already computed $M_{k-1}=$ $\min \left(s_{1}^{+}, \ldots, s_{k-1}^{+}\right)$, then we can compute $M_{k}$ as $M_{k}=\min \left(M_{k-1}, s_{k}^{+}\right)$(and $m_{k}$ as $\left.m_{k}=\max \left(m_{k-1}, s_{k}^{-}\right)\right)$.

\section{How to Find Monotonicity Regions of a Function Defined With Interval Uncertainty: Algorithm}

Thus, to find the monotonicity segments, we can use the following algorithm. In this algorithm, we process the intervals $\mathbf{s}_{1}, \ldots, \mathbf{s}_{n}$ one by one.

When we have the 1-st interval, then the only information that we have about the function $s(y)$ is that $s\left(y_{1}\right) \in \mathbf{s}_{1}$. This information is consistent with the function $s(y)$ being a constant, i.e., both non-decreasing and nonincreasing. Thus, we are still consistent with monotonicity. To start the process of computing $m_{k}$ and $M_{k}$, we assign $m_{1}:=s_{1}^{-}$and $M_{1}:=s_{1}^{+}$.

If the first $k$ intervals $\mathbf{s}_{k}$ are consistent with the assumption that the function $s(y)$ is non-decreasing, then when we get the new interval $\mathbf{s}_{k+1}$, we check whether $m_{k} \leq s_{k+1}^{+}$. Then: 
- If $m_{k} \leq s_{k+1}^{+}$, then the new point $y_{k+1}$ is still within the same monotonicity region. To prepare for the next interval, we compute $m_{k+1}=$ $\min \left(m_{k}, s_{k+1}^{-}\right)$.

- If $m_{k}>s_{k+1}^{+}$, this means that the new point $y_{k+1}$ cannot be within the same monotonicity region, and so the monotonicity region must end before $y_{k+1}$. The point $y_{k+1}$ itself belongs to a different monotonicity region which may expand both to the previous values $y_{k}, y_{k-1}, \ldots$, and to the following values $y_{k+2}, \ldots$; this new region can be traced in the same manner.

Similarly, if the first $k$ intervals $\mathbf{s}_{k}$ are consistent with the assumption that the function $s(y)$ is non-increasing, then when we get the new interval $\mathbf{s}_{k+1}$, we check whether $M_{k} \geq s_{k+1}^{-}$. Then:

- If $M_{k} \geq s_{k+1}^{-}$, then the new point $y_{k+1}$ is still within the same monotonicity region. To prepare for the next interval, we compute $M_{k+1}=$ $\max \left(M_{k}, s_{k+1}^{+}\right)$.

- If $M_{k}<s_{k+1}^{-}$, this means that the new point $y_{k+1}$ cannot be within the same monotonicity region, and so the monotonicity region must end before $y_{k+1}$. The point $y_{k+1}$ itself belongs to a different monotonicity region which may expand both to the previous values $y_{k}, y_{k-1}, \ldots$, and to the following values $y_{k+2}, \ldots$; this new region can be traced in the same manner.

\section{How to Find Monotonicity Regions of a Function Defined With Interval Uncertainty: Example}

Let us illustrate the above algorithm on the Rio Grande rift example.

We start with the 1 -st interval, for which $m_{1}:=s_{1}^{-}=0.26$ and $M_{1}:=$ $s_{1}^{+}=0.30$.

For the 2-nd interval, we check the inequalities $0.225=s_{2}^{-} \leq M_{1}=0.30$ and $0.255=s_{2}^{+} \geq m_{1}=0.26$. The first inequality holds, but the second does not hold, so we are in a non-increasing region. Thus, we compute $M_{2}=$ $\min \left(M_{1}, s_{2}^{+}\right)=\min (0.30,0.255)=0.255$.

For the 3-rd interval, the inequality $0.195=s_{3}^{-} \leq M_{2}=0.255$ still holds, so we are still in the non-increasing region. To prepare for the next interval, we compute $M_{3}=\min \left(M_{2}, s_{3}^{+}\right)=\min (0.255,0.225)=0.225$.

For the 4 -th interval, the inequality $0.14=s_{4}^{-} \leq M_{3}=0.225$ still holds, so we compute $M_{4}=\min \left(M_{3}, s_{4}^{+}\right)=\min (0.225,0.18)=0.18$.

For the 5 -th interval, the inequality $0.18=s_{4}^{-} \leq M_{4}=0.18$ still holds, so we compute $M_{5}=\min \left(M_{4}, s_{5}^{+}\right)=\min (0.18,0.22)=0.18$.

For the 6-th interval, the inequality $0.28=s_{5}^{-} \leq M_{5}=0.18$ no longer holds, so the first monotonicity region cannot continue past $y_{6}=34$. Thus, the first monotonicity region must be within $[29,34]$. 
The point $y_{6}$ must belong to the new monotonicity region, where the function $s(y)$ is non-decreasing instead of non-increasing. Before we go forward and start checking on the points $y_{7}$, etc., we must go back and check which points $y_{5}, y_{4}, \ldots$, can belong to this new region. If we have already checked that $y_{6}, \ldots, y_{k}$ belong to this region, this means that $s_{i}^{-} \leq s_{j}^{+}$for all such $i<j$. To check whether $y_{k-1}$ belongs to this same region, we must check whether $s_{k-1}^{-} \leq s_{i}^{+}$for all $\operatorname{such} i$, i.e., whether $s_{k-1}^{-} \leq \widetilde{M}_{k}=\min \left(s_{6}^{+}, \ldots, s_{k}^{+}\right)$.

We start with $\widetilde{M}_{6}=s_{6}^{+}=0.30$. Since the inequality $0.16=s_{5}^{-} \leq \widetilde{M}_{6}=$ 0.30 holds, $y_{5}$ also belongs to this region, so we compute $\widetilde{M}_{5}=\min \left(\widetilde{M}_{6}, s_{5}^{+}\right)=$ $\min (0.30,0.22)=0.22$.

For the 4 -th value, the inequality $0.14=s_{4}^{-} \leq \widetilde{M}_{5}=0.22$ holds, so $y_{4}$ also belongs to this region, and we compute $\widetilde{\widetilde{M}}_{4}=\min \left(\widetilde{M}_{5}, s_{4}^{+}\right)=$ $\min (0.22,0.18)=0.18$.

For the 3 -rd value, the inequality $0.195=s_{3}^{-} \leq \widetilde{M}_{4}=0.18$ is not true, so the new region must stop before $y_{3}=31$. Thus, the new region start at 31 .

Now, we can move forward and check whether points $y_{7}, \ldots$, belong to the new monotonicity region.

We start with $m_{6}:=s_{6}^{-}=0.28$. On the next step, we check whether $0.32=s_{7}^{+} \geq m_{6}=0.28$, and since this inequality holds, we compute $m_{7}=$ $\max \left(m_{6}, s_{7}\right)=\max (0.28,0.30)=0.30$. Similarly, we compute $m_{8}=0.33$, $m_{9}=0.405, m_{10}=0.80, m_{11}=0.80$, and $m_{12}=0.88$. For the 13-th interval, the inequality $0.85=s_{13}^{+} \geq m_{12}=0.88$ is no longer true, so $y_{13}$ cannot belong to the second (non-decreasing) monotonicity region. Thus, the second region must stop before $y_{13}=41$, and the point $y_{13}$ must belong to the third monotonicity region.

To find out which other points belong to this third region, we must go back and check which points $y_{12}, y_{11}, \ldots$, can belong to this new region. If we have already checked that $y_{13}, \ldots, y_{k}$ belong to this region, this means that $s_{i}^{+} \geq s_{j}^{-}$for all such $i<j$. To check whether $y_{k-1}$ belongs to this same region, we must check whether $s_{k-1}^{+} \geq s_{i}^{-}$for all such $i$, i.e., whether $s_{k-1}^{+} \geq \widetilde{m}_{k}=\max \left(s_{13}^{-}, \ldots, s_{k}^{-}\right)$.

We start with $\widetilde{m}_{13}=s_{13}^{-}=0.63$. Since the inequality $1.04=s_{12}^{+} \geq$ $\widetilde{m}_{13}=0.63$ holds, $y_{12}$ also belongs to this region, so we compute $\widetilde{m}_{12}=$ $\max \left(\widetilde{m}_{13}, s_{12}^{-}\right)=\max (0.63,0.88)=0.88$.

For the 11-th value, the inequality $0.88=s_{11}^{+} \geq \widetilde{m}_{12}=0.88$ holds, so $y_{11}$ also belongs to this region, and we compute $\widetilde{m}_{11}=\max \left(\widetilde{m}_{12}, s_{11}^{-}\right)=$ $\max (0.88,0.72)=0.88$.

For the 10 -th value, the inequality $1.10=s_{10}^{+} \geq \widetilde{m}_{11}=0.88$ holds, so $y_{10}$ also belongs to this region, and we compute $\widetilde{m}_{10}=\max \left(\widetilde{m}_{11}, s_{10}^{-}\right)=$ $\max (0.88,0.80)=0.88$.

For the 9-th value, the inequality $0.515=s_{9}^{+} \geq \widetilde{m}_{10}=0.88$ is not true, so the new region must stop before $y_{9}=37$. Thus, the new region starts at 37 . 
Thus, we have three monotonicity regions: $[29,34]$, [31, 41], and [37, 41]. The fact that we have discovered exactly three monotonicity regions is in good accordance with the geochemical data from [5].

\section{Comment: What Happens When We Use Shorter Wavelengths}

We ran the same algorithm for the spectral data corresponding to shorter wavelengths, i.e., for wavelengths $350-1000 \mathrm{~km}, 150-350 \mathrm{~km}$, etc. Intuitively, the shorter the wavelength, the more small-size structures (which are irrelevant for our large-scale subdivision) influence the spectral values. In full accordance with this expectation: for $350-1000$ and $150-350 \mathrm{~km}$, we got a similar classification, but with a much larger uncertainty; for even shorted wavelengths, we got a subdivision to many more zones which probably reflect some short-scale structures that we ignore in our subdivision into segments.

\section{From Intervals to Fuzzy}

For each segment $Y_{i}$ and for each point $y$, the algorithm tells whether a point $y$ can (in view of the data) belong to the segment $Y_{i}$ or not.

Some points belong to only one segment and therefore, are guaranteed to belong to this segment.

In the Rio Grande rift example, such are the points from the interval $\widetilde{Y}_{1}=[29,31]$ for the first region, the points from the interval $\widetilde{Y}_{2}=[34,37]$ for the second region, and the point $\widetilde{Y}_{3}=\{41\}$ for the third region.

Other points may belong to two neighboring segments $Y_{1}$ and $Y_{2}$, and the existing information is not sufficient to definitely conclude whether the given point $y$ belongs to the segment $Y_{1}$ or to the segment $Y_{2}$.

In particular, for all the points which can belong to two neighboring segments $Y_{1}$ and $Y_{2}$, the result of the above algorithm is the same. Thus, from the interval computations viewpoint, for all points $y$ which have not been uniquely classified, there is the same uncertainty. Intuitively, however, there is a reason to assume that we are more uncertainty about the classification of some of these points and less uncertainty about the classification of some other points.

For example, if a point $y$ is close to the set $\widetilde{Y}_{1}=\left[y_{1}^{-}, y_{1}^{+}\right]$of all points which are proven to belong to $Y_{1}$, and it is distant from the set $\widetilde{Y}_{2}=\left[y_{2}^{-}, y_{2}^{+}\right]$ of all the points which are proven to belong to $Y_{2}$, then, intuitively, it seems reasonable to conclude that the point $y$ is more probably to be in the segment $Y_{1}$. Similarly, $y$ is close to $\widetilde{Y}_{2}$ and distant from $\widetilde{Y}_{1}$, then, intuitively, it seems reasonable to conclude that the point $y$ is more probably to be in the segment $Y_{2}$. 
This intuitive sense can be captured if, instead of simply describing which points $y$ can be in which segments, we produce, for each segment $Y_{i}$ and for each $y$, not only the values "true" or "false" indicating that the point $y$ can or cannot belong to $Y_{i}$, but also the "degree" $\mu_{i}(y)$ to which $y$ can belong to the segment $Y_{i}$. For two neighboring segments $Y_{1}$ and $Y_{2}$, the degree $\mu_{1}(y)$ must be equal to 1 for $y \in \widetilde{Y}_{1}=\left[y_{1}^{-}, y_{1}^{+}\right]$and to 0 for $y \in \widetilde{Y}_{2}=\left[y_{2}^{-}, y_{2}^{+}\right]$. It therefore makes sense to use a linear extrapolation to determine the values of the function $\mu_{1}(y)$ for $y \in\left[y_{1}^{+}, y_{2}^{-}\right]$. Linear extrapolation is widely (and successfully) used in applications of fuzzy techniques (see, e.g., $[4,7]$ ), and it is also known that linear extrapolation is (in some reasonable sense) the most robust extrapolation procedure (see, e.g., [6]).

As a result, for each segment $Y_{i}$, the resulting membership function $\mu_{i}(y)$ is trapezoidal:

- $\mu_{i}(y)=0$ for $y \leq y_{i-1}^{+}$;

- $\mu_{i}(y)=\frac{y-y_{i-1}^{+}}{y_{i}^{-}-y_{i-1}^{+}}$for $y \in\left[y_{i-1}^{+}, y_{i}^{-}\right]$,

- $\mu_{i}(y)=1$ for $y \in\left[y_{i}^{-}, y_{i}^{+}\right]$

- $\mu_{i}(y)=\frac{y_{i+1}^{-}-y}{y_{i+1}^{-}-y_{i}^{+}}$for $y \in\left[y_{i}^{+}, y_{i+1}^{-}\right]$, and

- $\mu_{i}(y)=0$ for $y \geq y_{i+1}^{-}$.

\section{Acknowledgments}

This work was supported in part by NASA under cooperative agreement NCC5-209, by NSF grants No. DUE-9750858 and CDA-9522207, by the United Space Alliance, grant No. NAS 9-20000 (PWO C0C67713A6), by the Future Aerospace Science and Technology Program (FAST) Center for Structural Integrity of Aerospace Systems, effort sponsored by the Air Force Office of Scientific Research, Air Force Materiel Command, USAF, under grant number F49620-95-1-0518, and by the National Security Agency under Grant No. MDA904-98-1-0561.

\section{References}

1. Höppner, F. (1999) Fuzzy Cluster Analysis. J. Wiley, New York.

2. Jain, A. K., Dubes, R. C. (1988) Algorithms for Clustering Data. Prentice Hall, Englewood Cliffs, NJ.

3. Kearfott, R. B., Kreinovich, V., eds. (1996) Applications of Interval Computations, Kluwer, Dordrecht.

4. Klir, G., Yuan, B. (1995) Fuzzy Sets and Fuzzy Logic: Theory and Applications, Prentice Hall, Upper Saddle River, NJ.

5. N. J. McMillan, N. J. (1998) Temporal and spatial magmatic evolution of the Rio Grande rift. New Mexico Geological Survey Guidebook, 49th Field Conference, Las Cruces County, 107-116. 
6. Nguyen, H. T., Kreinovich, V., Lea, B., Tolbert, D. (1995) Interpolation that leads to the narrowest intervals, and its application to expert systems and intelligent control. Reliable Computing 1, No. 3, 299-316.

7. Nguyen, H. T., Walker, E. A. (1999) First Course in Fuzzy Logic, CRC Press, Boca Raton, FL.

8. Villaverde, K., and Kreinovich, V. (1993) A linear-time algorithm that locates local extrema of a function of one variable from interval measurement results. Interval Computations, No. 4, 176-194. 